\title{
Rotational Atherectomy Followed by Drug-Coated Balloon Dilation in Possible Coronary Sequelae of Kawasaki Disease
}

\author{
Jun Shiraishi, ${ }^{1}$ MD, Yuki Matsubara, ${ }^{1}$ MD, Takashi Yanagiuchi, ${ }^{1}$ MD, Akira Shikuma, ${ }^{1}$ MD, \\ Keisuke ShoJI, ${ }^{1}$ MD, Marie NishiKawa, ${ }^{1}$ MD, Daisuke ITo, ${ }^{1}$ MD, Masayoshi Kimura, ${ }^{1}$ MD, \\ Eigo Kishita, ${ }^{1}$ MD, Yusuke NaKagawa, ${ }^{1}$ MD, Masayuki Hyogo, ${ }^{1}$ MD, \\ Takahisa SAwAdA, ${ }^{1} \mathrm{MD}$, and Yoshio KoHNO, ${ }^{1} \mathrm{MD}$
}

\begin{abstract}
SUMMARY
Rotational atherectomy with/without low-pressure balloon dilation has been a mainstay of interventional treatment for stenosis due to the coronary sequelae of Kawasaki disease (KD). Here, we report a restenosis case of probable coronary sequelae of $\mathrm{KD}$ treated with rotational atherectomy with low-pressure 2.5-mm balloon dilation 6 months previously. Under the guidance of optical frequency domain imaging, we performed rotational atherectomy followed by $2.5-\mathrm{mm}$ drug-coated balloon (DCB) dilation for an atherosclerotic restenosis at the inlet of a calcified aneurysm in the proximal left anterior descending coronary artery. Coronary angiography 6 months later showed no apparent progression of vessel narrowing, and we could defer repeat intervention. The present case suggests that rotational atherectomy followed by DCB dilation could be an alternative revascularization therapy of choice in coronary KD sequelae complicated with atherosclerosis. (Int Heart J 2016; 57: 367-371)
\end{abstract}

Key words: Optical frequency domain imaging, Fractional flow reserve, Atherosclerosis, Dyslipidemia

$\mathrm{C}$ oronary sequelae of Kawasaki disease (KD) has a variety of morphological manifestations, such as aneurysm, stenosis, and total occlusion concomitant with nonatherosclerotic intimal thickening and frequent severe calcification, while a few reports have demonstrated early atherosclerosis progression in young adults with prior $\mathrm{KD}^{1-3)}$ Since these stiff calcified lesions have risk of stent underexpansion and neo-aneurysmal formation ascribed to high-pressure balloon inflation, rotational atherectomy with/without additional low-pressure balloon dilation alone has been a mainstay of interventional treatment for stenosis due to the coronary sequelae of $\mathrm{KD}^{4}{ }^{4 \cdot 6)}$ In contrast, drug-coated balloons (DCB) now play a central role in the treatment of in-stent restenosis through their inhibition of neointimal hyperplasia, despite the lack of evidence for de novo coronary artery disease. ${ }^{7.8)}$ We describe here our experience with an atherosclerotic-restenosis case of probable coronary sequelae of KD previously treated with rotational atherectomy with additional low-pressure balloon dilation 6 months before, ${ }^{3)}$ in which rotational atherectomy followed by DCB dilation could defer any subsequent repeat intervention.

\section{CASe Report}

An asymptomatic 47 year-old male was admitted to our hospital to undergo 6-month follow-up coronary angiography
(CAG). Six months previously, based on a diagnosis of silent myocardial ischemia due to possible sequelae of $\mathrm{KD}$, we had performed rotational atherectomy with $1.5 / 2.0 \mathrm{~mm}$ burrs followed by low-pressure dilation using a balloon catheter 2.5/15 $\mathrm{mm}$ at $4 \mathrm{~atm}$ for a stenosis at the inlet of a calcified aneurysm in the proximal segment of the left anterior descending coronary artery (LAD) (Figure 1A, 1B). Although he had no apparent history of $\mathrm{KD}$, he was hospitalized for 40 days due to fever of unknown cause with systemic eruption at the age of 6 months. His coronary risk factor was dyslipidemia, and under medication consisting of rosuvastatin $10 \mathrm{mg} /$ day, ezetimibe 10 $\mathrm{mg} / \mathrm{day}$, and fenofibrate $80 \mathrm{mg} / \mathrm{day}$, his blood test at admission showed low-density lipoprotein of $131 \mathrm{mg} / \mathrm{dL}$, high-density lipoprotein of $55 \mathrm{mg} / \mathrm{dL}$, and triglycerides of $103 \mathrm{mg} / \mathrm{dL}$. Electrocardiogram at admission was normal, and echocardiography showed no asynergy with a left ventricular ejection fraction of $75 \%$. Six-month follow-up CAG showed an intermediate stenosis at the culprit site (Figure 1C), and subsequent fractional flow reserve (FFR) measurement revealed 0.72 at the mid LAD distal to the aneurysm (intra-aneurysm 0.75 , LAD proximal to the aneurysm 1.00). During the prior percutaneous coronary intervention (PCI), optical frequency domain imaging (OFDI) revealed fibrofatty plaque with segmental calcification (Figure 3A) at the inlet of the calcified aneurysm, suggesting that repeat rotational atherectomy followed by low-pressure "bare" balloon dilation alone could not prevent re-restenosis

From the ${ }^{1}$ Department of Cardiology, Kyoto First Red Cross Hospital, Kyoto, Japan.

Address for correspondence: Jun Shiraishi, MD, Department of Cardiology, Kyoto First Red Cross Hospital, Honmachi, Higashiyama-ku, Kyoto 605-0981, Japan. E-mail: risa11221998@yahoo.co.jp

Received for publication September 7, 2015. Revised and accepted November 16, 2015.

Released in advance online on J-STAGE April 28, 2016.

All rights reserved by the International Heart Journal Association. 

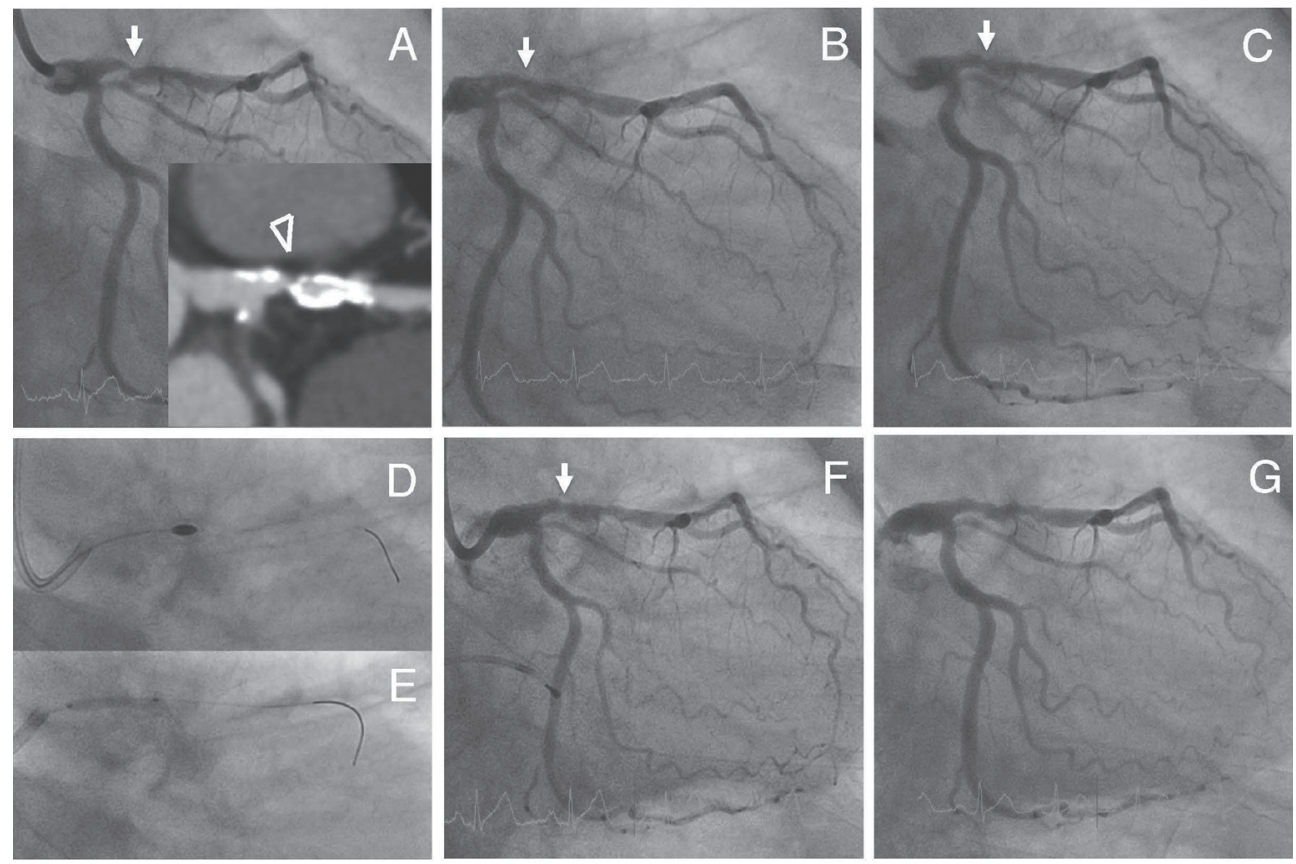

Figure 1. Right anterior caudal images of left coronary angiography (CAG). A: CAG and coronary CT before first PCI Magnified image of curved multiplanar reconstruction image of the coronary CT depicted stenosis composed of low-density plaque and calcification (white arrowhead) at the inlet of the eggshell-like severely calcified aneurysm. B: Post first PCI (rotational atherectomy with a $2.0 \mathrm{~mm}$ burr followed by low-pressure $2.5-\mathrm{mm}$ balloon dilation). C: Six months after the first PCI. D: Ablation with a $2.15 \mathrm{~mm}$ burr in the proximal LAD. E: Adjunctive 2.5 - $\mathrm{mm}$ drug-coating balloon dilation in the proximal LAD. F: Post second PCI. G: Six months after the second PCI. (A and B from Shiraishi, et $a l,{ }^{3)}$ copyright the Japanese Association of Cardiovascular Intervention and Therapeutics, reprinted with permission)
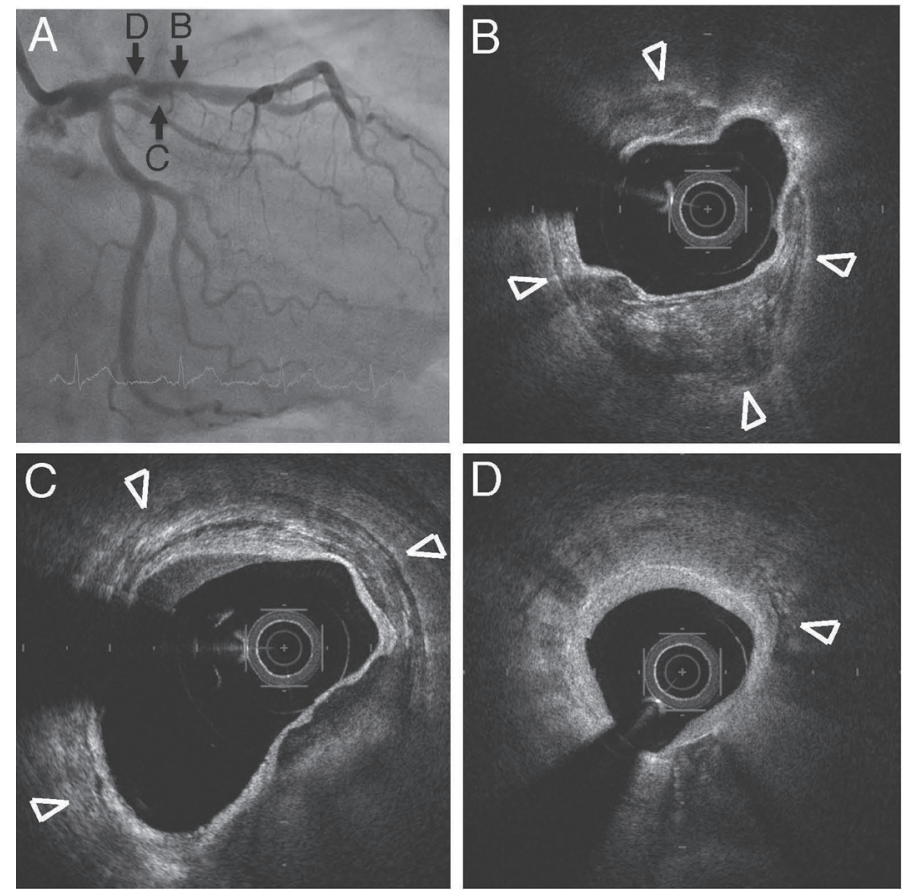

Figure 2. Right anterior caudal view of left coronary angiography (A) and optical frequency domain imaging images (B-D, corresponding to $\mathbf{A}$ [black arrows], respectively) before the second PCI. Almost circumferential thick calcification at the outlet of the aneurysm (B, white arrowheads) as well as at the aneurysm (C, white arrowheads). D: Newly formed concentric layer with homogeneous high intensity signal on the lumen-side of preexisting intima and focal calcification (white arrowhead) at the inlet of the aneurysm. 

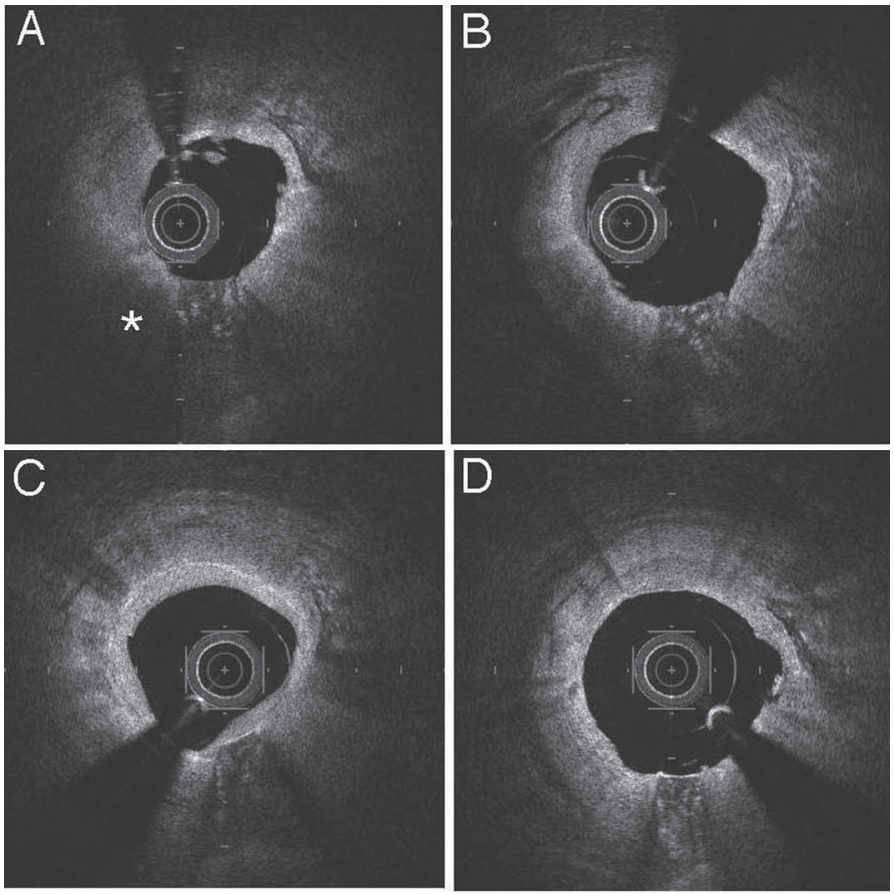

Figure 3. Optical frequency domain imaging (OFDI) images of the culprit lesion at the inlet of the aneurysm. A: OFDI image after the ablation with a $1.5 \mathrm{~mm}$ burr during the first PCI. Fibrofatty plaque was detected (white star). B: OFDI image after the first PCI (rotational atherectomy with a $2.0 \mathrm{~mm}$ burr followed by low-pressure 2.5 - $\mathrm{mm}$ balloon dilation), corresponding to Figure 1B (white arrow). Minimum lumen area (MLA) $3.3 \mathrm{~mm}^{3}$. C: OFDI image before the second PCI, corresponding to Figure 1C (white arrow). MLA $2.4 \mathrm{~mm}^{3}$. D: OFDI image after the second PCI (rotational atherectomy with a $2.15 \mathrm{~mm}$ burr followed by $2.5-\mathrm{mm}$ drug-coating balloon dilation), corresponding to Figure $1 \mathrm{~F}$ (white arrow). MLA 3.5 $\mathrm{mm}^{3}$. (A and $\mathbf{B}$ from Shiraishi, et al, ${ }^{3)}$ copyright the Japanese Association of Cardiovascular Intervention and Therapeutics, reprinted with permission)

because of the concomitant atherosclerotic change at the inlet. In addition, drug-eluting stent (DES) implantation with highpressure dilation might provoke neoaneurysmal formation at the culprit and stent malapposition at the aneurysm. Taking these risks of neoaneurysmal formation and recurrent restenosis into consideration, we therefore decided to perform transfemoral PCI for the restenosis lesion in the proximal LAD, using rotational atherectomy with a larger burr and additional balloon angioplasty with DCB under the guidance of OFDI as well as under dual antiplatelet therapy (aspirin $100 \mathrm{mg} /$ day and clopidogrel $75 \mathrm{mg} /$ day) to minimize vessel-wall damage and intimal proliferation.

An 8-Fr long sheath and a 6-Fr sheath were introduced into his right femoral artery and right femoral vein, respectively, and 7,000 $\mathrm{U}$ of unfractionated heparin was administered through the right femoral vein sheath. Using an 8-Fr guiding catheter (mach-1, Q 3.5 SH, Boston Scientific), a guidewire (Sion blue, Asahi Intecc) was passed through the culprit lesion in the proximal LAD and advanced into the distal LAD. OFDI (Fastview, TERUMO) (Figure 2A-2D) revealed fibrous intimal proliferation characterized by a homogeneous high intensity signal at the inlet of the aneurysm (Figure 2D) as well as surrounding calcification at the aneurysm (Figure 2C) including the outlet (Figure 2B). Based on the OFDI findings and the step-up site on the prior FFR measurement, we decided to perform re-PCI solely for the inlet of the aneurysm in the proximal LAD. We then exchanged the wire for another wire (Ro- tawire floppy, Boston Scientific) via a micro catheter (Finecross GT, TERUMO), and performed rotational atherectomy with a $2.15 \mathrm{~mm}$ burr (Rotalink Plus, Boston Scientific, initial 160,000 rpm, final 190,000 rpm). Since we could not pass the burr through the inlet, we exchanged the burr for a downsized burr $(2.00 \mathrm{~mm})$ and performed an ablation $(200,000$ rpm). Next, we added rotational atherectomy with a $2.15 \mathrm{~mm}$ burr (200,000 rpm) (Figure 1D) and dilation using a DCB catheter (SeQuent Please, 2.5/15 mm, B. Braun) at 7 atm to treat the residual stenosis (Figure 1E). The final CAG showed an acceptable result (Figure 1F), and the final OFDI showed no apparent dissection or cracks (Figure 3D). Follow-up CAG 6 months after the last PCI showed a mild stenosis at the culprit site (Figure 1G), and subsequent FFR measurement revealed 0.76 at the mid LAD distal to the aneurysm. Taking account of the asymptomatic status, FFR value $>0.75$, clinical course and disease nature, we decided to defer repeat PCI and the patient has been kept free of cardiac events under conventional medical treatment (aspirin and anti-dyslipidemic agents).

\section{DisCUSSION}

In the coronary sequelae of $\mathrm{KD}$, aneurysmal formation tends to involve proximal portions of the coronary tree, and often regress concomitant with occasional narrowing particularly at the inlet or the outlet of the aneurysm over the long-term. 
Regressed aneurysms show marked intimal thickening with or without calcification particularly at the inlet or the outlet, not uncommonly leading to coronary stenosis or occlusion. ${ }^{9,10)}$ Coronary KD sequelae is one of the underlying diseases of coronary events in young adults. ${ }^{11,12)}$ The present case also showed moderate intimal thickening with surrounding calcification at the aneurysm in the proximal LAD accompanied with lumen narrowing at the inlet on OFDI, and the aneurysm could not be delineated by CAG alone. In contrast, coronary computed tomography (CT) before the first PCI depicted eggshelllike calcified aneurysm in the proximal LAD and enlargement in the left main trunk and in the just proximal LAD, in which vessel diameters at the aneurysm and the distal reference were $6.1 \mathrm{~mm}$ and $3.5 \mathrm{~mm}$, respectively, while no definite plaques were observed in the mid-distal LAD, right coronary artery, or left circumflex coronary artery. ${ }^{3)}$ In addition, previous reports have demonstrated that patients with coronary aneurysms who do not fulfill the diagnostic criteria of KD might have had antecedent incomplete or atypical KD. ${ }^{13,14)}$ The present case had no apparent history of $\mathrm{KD}$, but had a history suggesting KD or incomplete $\mathrm{KD}$, such as fever of unknown cause with systemic eruption at the age of 6 months, even though the disease concept of KD was not established at that time. Moreover, the distribution of calcification and the wall structure in the culprit artery on coronary $\mathrm{CT}$ and OFDI/intravascular ultrasound (IVUS) during the first PCI closely resembled those ascribed to KD sequelae complicated with atherosclerosis. ${ }^{3)}$ We have therefore speculated that the patient had suffered from KD or incomplete KD in his infancy, and had silent myocardial ischemia due to coronary KD sequelae complicated with atherosclerosis in adulthood.

Coronary sequelae of $\mathrm{KD}$, composed of dense fibrosis and calcification, is generally stiff, and high-pressure balloon dilatation as well as stent implantation with high-pressure can lead to late neo-aneurysmal formation in KD sequelae. ${ }^{4-6)}$ Rotational atherectomy with/without additional low-pressure balloon dilation alone therefore has been a standard interventional strategy for coronary stenosis due to KD sequelae, ${ }^{4-6)}$ and usage of larger sized burrs is associated with a lower frequency of restenosis. ${ }^{15)}$ In the present case, the culprit lesion itself at the inlet of the aneurysm had mainly fibrous intimal thickening with localized calcification on OFDI during the second PCI, while the aneurysm had severe eggshell-like calcification. Thus, it might not be absolutely necessary to perform rotational atherectomy, however, in order to avoid DES implantation requiring high-pressure inflation, we performed rotational atherectomy with a larger burr as lesion debulking rather than lesion modification.

PCI mainly using rotational atherectomy for KD sequelae has been reported to have lesser prevalence of restenosis on CAG and post-procedural intimal hyperplasia on IVUS than PCI for atherosclerotic lesions in adults. ${ }^{4-6)}$ The mechanism responsible for this finding is unclear, however, differences in tissue composition of vessel walls might play a role. Thickened intima in KD coronary sequelae is generally composed of fibrous tissue with lower cell density, inconsistent with adult atherosclerotic plaque, ${ }^{1)}$ implying lower proliferative properties of intima in KD sequelae in response to PCI-related vessel injury. In the present case, however, during the first PCI, OFDI delineated fibrofatty plaque at the culprit site, indicating the presence of early atherosclerotic change in KD sequelae. ${ }^{3)}$
Thus, this culprit site, even though due to KD sequelae, might have a relatively high probability of restenosis. Indeed, OFDI 6 months after the first PCI depicted a newly formed concentric layer with a homogeneous high intensity OFDI signal on the lumen-side of preexisting intima, suggesting that neointimal proliferation consisting of dense fibrosis and accumulation of smooth muscle cells might play a crucial role in the restenosis process, consistent with usual post-procedural intimal hyperplasia in atherosclerotic lesions. ${ }^{16)}$

As a first-line interventional treatment for in-stent restenosis during the DES era, the usefulness of DCB has almost been established. ${ }^{7,8)}$ In contrast, for de novo coronary artery lesions, no definite evidence supporting the superiority of DCB over DES is available, ${ }^{17,18)}$ while a few registries have shown acceptable results regarding the safety and efficacy of DCB in de novo coronary lesions. ${ }^{19,20)}$ In the Valentines II, a prospective registry of 109 lesions in 103 patients with stable or unstable angina and/or positive stress test with de novo lesions of $>$ $50 \%$ stenosis undergoing plain old balloon angioplasty followed by DCB dilation, the primary endpoint of major adverse cardiac events at 8 months was $8.7 \%$ with $1 \%$ all-cause death, $1 \%$ myocardial infarction, and $2.9 \%$ target lesion revascularization (TLR), despite the $11.9 \%$ bail-out bare metal stent (BMS) implantation. ${ }^{19)}$ In part of the SeQuent Please World Wide Registry, a prospective registry of 554 lesions in 496 patients with de novo lesions (small vessels with a mean reference diameter of $2.6 \mathrm{~mm}$ ) undergoing DCB dilation alone or DCB dilation followed by BMS implantation, the primary endpoint of clinically driven TLR at 9 months was $1.0 \%$ in DCB alone and $2.4 \%$ in DCB/BMS with $0.7 \% / 0.0 \% \mathrm{MI}$ and $1.0 \% / 1.2 \%$ cardiac death, respectively. ${ }^{20)}$ Although the role of DCB in de novo coronary artery lesions including post-rotational atherectomy lesions remains unclear, it is reasonable to propose that DCB-based PCI could be an alternative revascularization therapy of choice among patients with de novo coronary lesions who are inappropriate candidates for DES implantation. With regard to the coronary sequelae of $\mathrm{KD}$, because of probable differences in the proliferative properties of intima in response to PCI-related vessel injury between adult atherosclerotic lesions and conventional KD sequelae as described above, there is a possibility that DCB might confer more effective anti-restenotic properties, particularly on coronary KD sequelae complicated with atherosclerosis rather than conventional coronary KD sequelae. In the present case, however, not only additional dilation with DCB but also ablation by larger-sized burrs might contribute to deferring repeat PCI. This is the first report to describe performing rotational atherectomy followed by $\mathrm{DCB}$ dilation in the coronary sequelae of $\mathrm{KD}$.

According to the JCS guidelines, IVUS usage is recommended during PCI for the coronary sequelae of KD. ${ }^{21)}$ However, in those cases with severe calcification like the present case, it is difficult to evaluate plaque distribution/characterization except for calcification as well as the precise depth of calcification by gray-scale IVUS. Indeed, in the present case, we used both IVUS and OFDI, a new generation of optical coherence tomography (OCT) as imaging modalities during the first PCI, and it was impossible to evaluate plaque morphology or the precise extent/depth of calcification at the aneurysm by gray-scale IVUS, but it was possible to do so by OFDI. ${ }^{3}$ Moreover, OCT has a potential to clearly delineate inner intimal proliferation as layered intima by its high image resolu- 
tion, consistent with the present report. ${ }^{22)}$ Nevertheless, there are only limited data regarding OCT findings in post-KD patients, in which concentric/eccentric intimal thickening, calcification, disappearance of the media, white thrombus, neo-vascularization, and lipid deposition have been observed. ${ }^{3,23,24)}$ Thus, it is reasonable to propose that not only IVUS but also OFDI might be a useful imaging device, especially for PCI against complex lesions such as the coronary sequelae of $\mathrm{KD}$. Conclusion: The present findings suggest that rotational atherectomy followed by DCB dilation could be an alternative revascularization therapy of choice in coronary $\mathrm{KD}$ sequelae complicated with atherosclerosis.

\section{Disclosure}

Conflict of interest: The authors have no conflicts of interest to report regarding the content of the manuscript.

\section{REFERENCES}

1. Takahashi K, Oharaseki T, Naoe S. Pathological study of postcoronary arteritis in adolescents and young adults: with reference to the relationship between sequelae of Kawasaki disease and atherosclerosis. Pediatr Cardiol 2001; 22: 138-42.

2. Negoro N, Nariyama J, Nakagawa A, et al. Successful catheter interventional therapy for acute coronary syndrome secondary to kawasaki disease in young adults. Circ J 2003; 67: 362-5.

3. Shiraishi J, Yashige M, Hyogo M, Shima T, Sawada T, Kohno Y Lipid-rich plaque in possible coronary sequelae of Kawasaki disease detected by optical frequency domain imaging. Cardiovasc Interv Ther 2015; 30: 367-71.

4. Sugimura T, Yokoi H, Sato N, et al. Interventional treatment for children with severe coronary artery stenosis with calcification after long-term Kawasaki disease. Circulation 1997; 96: 3928-33.

5. Akagi T, Ogawa S, Ino T, et al. Catheter interventional treatment in Kawasaki disease: A report from the Japanese Pediatric Interventional Cardiology Investigation group. J Pediatr 2000; 137: 181-6.

6. Ishii M, Ueno $\mathrm{T}$, Ikeda $\mathrm{H}$, et al. Sequential follow-up results of catheter intervention for coronary artery lesions after Kawasaki disease: quantitative coronary artery angiography and intravascular ultrasound imaging study. Circulation 2002; 105: 3004-10.

7. Loh JP, Waksman R. Paclitaxel drug-coated balloons: a review of current status and emerging applications in native coronary artery de novo lesions. JACC Cardiovasc Interv 2012; 5: 1001-12. (Review)

8. Byrne RA, Joner M, Alfonso F, Kastrati A. Drug-coated balloon therapy in coronary and peripheral artery disease. Nat Rev Cardiol 2014; 11: 13-23. (Review)

9. Sugimura $\mathrm{T}$, Kato $\mathrm{H}$, Inoue $\mathrm{O}$, et al. Intravascular ultrasound of coronary arteries in children. Assessment of the wall morphology and the lumen after Kawasaki disease. Circulation 1994; 89: 25865.

10. Kato H, Sugimura T, Akagi T, et al. Long-term consequences of Kawasaki disease. A 10- to 21-year follow-up study of 594 patients. Circulation 1996; 94: 1379-85.
11. Shiraishi J, Kohno Y, Sawada T, et al. Pathogenesis of acute myocardial infarction in young male adults with or without obesity. $\mathrm{J}$ Cardiol 2007; 49: 13-21.

12. Motozawa Y, Uozumi H, Maemura S, et al. Acute myocardial infarction that resulted from poor adherence to medical treatment for giant coronary aneurysm. Int Heart J 2015; 56: 551-4.

13. Kato H, Inoue O, Kawasaki T, Fujiwara H, Watanabe T, Toshima H. Adult coronary artery disease probably due to childhood Kawasaki disease. Lancet 1992; 340: 1127-9.

14. Newburger JW, Takahashi M, Gerber MA, et al; Committee on Rheumatic Fever, Endocarditis and Kawasaki Disease; Council on Cardiovascular Disease in the Young; American Heart Association; American Academy of Pediatrics. Diagnosis, treatment, and long-term management of Kawasaki disease: a statement for health professionals from the Committee on Rheumatic Fever, Endocarditis and Kawasaki Disease, Council on Cardiovascular Disease in the Young, American Heart Association. Circulation 2004; 110: 2747-71

15. Tsuda E, Miyazaki S, Yamada O, Takamuro M, Takekawa T, Echigo S. Percutaneous transluminal coronary rotational atherectomy for localized stenosis caused by Kawasaki disease. Pediatr Cardiol 2006; 27: 447-53.

16. Tearney GJ, Regar E, Akasaka T, et al; International Working Group for Intravascular Optical Coherence Tomography (IWGIVOCT). Consensus standards for acquisition, measurement, and reporting of intravascular optical coherence tomography studies: a report from the International Working Group for Intravascular Optical Coherence Tomography Standardization and Validation. J Am Coll Cardiol 2012; 59: 1058-72.

17. Cortese B, Micheli A, Picchi A, et al. Paclitaxel-coated balloon versus drug-eluting stent during PCI of small coronary vessels, a prospective randomised clinical trial. The PICCOLETO study. Heart 2010; 96: 1291-6.

18. Latib A, Colombo A, Castriota F, et al. A randomized multicenter study comparing a paclitaxel drug-eluting balloon with a paclitaxel-eluting stent in small coronary vessels: the BELLO (Balloon Elution and Late Loss Optimization) study. J Am Coll Cardiol 2012; 60: 2473-80

19. Waksman R, Serra A, Loh JP, et al. Drug-coated balloons for de novo coronary lesions: results from the Valentines II trial. Eurointervention 2013; 9: 613-9.

20. Wöhrle J, Zadura M, Möbius-Winkler S, et al. SeQuentPlease World Wide Registry: clinical results of SeQuent please paclitaxelcoated balloon angioplasty in a large-scale, prospective registry study. J Am Coll Cardiol 2012; 60: 1733-8.

21. JCS Joint Working Group. Guidelines for diagnosis and management of cardiovascular sequelae in Kawasaki disease (JCS 2013). Digest version. Circ J 2014; 78: 2521-62.

22. Imamura $\mathrm{T}$, Kinugawa $\mathrm{K}$, Murasawa $\mathrm{T}$, et al. Cardiac allograft vasculopathy can be distinguished from donor-transmitted coronary atherosclerosis by optical coherence tomography imaging in a heart transplantation recipient: double layered intimal thickness. Int Heart J 2014; 55: 178-80.

23. Harris KC, Manouzi A, Fung AY, et al. Feasibility of optical coherence tomography in children with Kawasaki disease and pediatric heart transplant recipients. Circ Cardiovasc Imaging 2014; 7 : 671-8.

24. Kakimoto N, Suzuki H, Kubo T, et al. Evaluation of coronary arterial lesions due to Kawasaki disease using optical coherence tomography. Can J Cardiol 2014; 30: 956.e7-9. 